\title{
The efficacy of different methods of teeth cleaning on the levels of volatile sulfur compounds in patients with orthodontic fixed appliances
}

\author{
AlAnoud Mishal AlHarbi ${ }^{1 *}$, Omar Al-Kadhi ${ }^{2}$ and Jamal Al-Sanea ${ }^{2}$ \\ ${ }^{1}$ Orthodontic Department, BDS, Riyadh Elm University, Saudi Arabia \\ ${ }^{2}$ Orthodontics, Riyadh Elm University, Saudi Arabia
}

\begin{abstract}
Aim: To determine the effect of different cleaning methods and devices in reducing halitosis during active orthodontic treatment.

Materials \& Methods: 39 patients were randomly allocated into 4 groups. Group A: Using manual tooth brush and dental floss. Group B: Using electric tooth brush and dental floss. Group C: Using manual tooth brush with water jet device. Group D: Using electrical tooth brush and water jet device. each group were underwent clinical evaluation and oral malodor assessment Each participant was examined at baseline (T1) and after 4-6 weeks (T2) and then after 8-12 weeks (T3). Portable halimeter was used to evaluate the level of Volatile Sulphur compound for each participant at each time point. Oral malodor values were divided into five categories and classified as normal (1), weak (2), medium (3) strong (4), or very strong (5).

Sample size: Sample size calculation has been done based on previous literature. The sample size was calculated as 39 participants.

Results: No clinically significant differences were found between the four groups in the Volatile Sulphur Compound level (VSC). Also there was no clinically significant differences between females and males.

Conclusion: Oral malodor could indicate a need to evaluate oral health and remind patients of the importance of maintaining ideal oral hygiene during fixed orthodontic treatment. When Comparing the efficacy of these four different methods of teeth cleaning on reducing VSC in patients with active orthodontic fixed appliances, results found that there are No significant difference between controlled group and the other groups.
\end{abstract}

\section{Introduction}

There is an increasing in number of patients who seeks orthodontic treatment in contemporary dental care to correct malocclusion and aesthetic dental problems that can cause negative physical, social and psychological effects. Nevertheless, after the placements of fixed orthodontic appliances, patients continue to deal with other struggles such as plaque accumulation leading to an increased risk of evolving gingivitis, white spot lesions and halitosis [1].

Dentofacial complex mainly affected by Orthodontic treatment. Therefore, it is broadly practiced branch of dentistry. A lot of people don't brush teeth regularly after main meals. Because of that, there is food accumulation. According to previous studies there is a rapid decrease in oral hygiene compliance after the initial bonding, and the appliance helps plaque build-up and it is an obstruction to the hygiene practice like tooth brushing and flossing. This can even initiate gingivitis. Enamel decalcification may occur due to plaque build-up around the bracket base. Orthodontic patients are instructed to rinse two times a day with $20 \mathrm{~mL}$ of mouthwashes as an adjunct to brushing and flossing.

Demineralization commonly follows fixed orthodontic treatment, if oral hygiene is not maintained. The reason for this is the bacteria's acidic deposits in the plaque and it has negative effect on aesthetics [1].

Orthodontic treatments are usually done for children and adolescents, which can be challenging as they tend to have fewer skills and pay less attention to their oral health \& hygiene.
A lot of factors affects oral health including oral hygiene measures such as the frequency of brushing, the type of toothpaste used, and the method of brushing and additional measures such as the use of mouthwash and interdental floss or brush. Eating habits, the role of parents, the social situation and the education of the patient are also important. Accordingly, the important role of orthodontists is to instruct children and their parents how to take care of their oral health [2].

For patients with fixed orthodontic appliances it is hard to achieve good oral hygiene because the appliances can be an barrier to mechanical brushing - food can repeatedly get trapped around the brackets and under the arch wires after eating, and for patients whose treatment is lengthy it can be a challenge to preserve good oral health and avoid enamel demineralization, periodontal disease or halitosis.

Atassi, et al. reported that after three months of orthodontic treatment beginning there is a statistically significant increase in stimulated salivary flow rate, buffer capacity and levels of lactobacilli, as well as increased bleeding on probing (BOP), a higher plaque index

*Correspondence to: AlAnoud Mishal AlHarbi, Orthodontic Department, BDS, Riyadh Elm University, Kingdom of Saudi Arabia, E-mail: alanoud.m.alharbi@ student.riyadh.edu.sa

Received: November 08, 2020; Accepted: November 16, 2020; Published: November 19, 2020 
(PI), a higher gingival index (GI), and an increase in probing pocket depth (PPD) [3].

There are several studies that evaluate the effects of orthodontic treatment on periodontal status. The brackets and ligatures have a negative effect on natural cleaning by producing retention areas for plaques, making the mechanical cleaning of the teeth and gingiva by the tongue and lips more difficult, and increasing the viscosity of the saliva. Periodontal inflammation, hyperemia, hyperplasia, and demineralization of the teeth have been found as a consequence of failure of ensuring adequate oral hygiene.

It has been stated that accumulation of plaque and the increase in bacterial count, and tongue coating noticeably are also involved in the formation of halitosis [1].

Lately, some investigators have reported that fixed orthodontic appliances can play a major role in increasing oral malodor, and that oral malodor should be regarded as one of prospective side effects of orthodontic treatments [4].

Human breath is composed of highly complex substances with numerous variable odors which can generate unpleasant situations like halitosis. Halitosis is a latin word which derived from halitus (breathed air) and the osis (pathologic alteration),and it is used to explain any offensive bad or unpleasant odor arising from the mouth air and breath.

This undesirable condition is a common complaint for both genders and for all age groups. It creates social and psychological disadvantages for individuals, and these circumstances affect individual's relation with other people [5].

\section{Aim of the study}

To determine the effect of different cleaning methods and devices in reducing halitosis during active orthodontic treatment.

\section{Hypothesis}

Orthodontic treatment can interfere with halitosis and different malocclusions can help in accumulation of dental plaque.

\section{Definitions of halitosis}

Halitosis is the general term used to describe a foul odor emanating from the oral cavity, in which proteolysis, metabolic products of the desquamating cells and bacterial putrefaction are involved [6].

Halitosis is the general term used to describe any disagreeable odor in expired air, regardless of whether the odorous substances originate from oral or non- oral sources [7].

Halitosis is also termed as fetor ex ore or fetor oris. It is a foul or offensive odor emanating from the oral cavity [8]

Unpleasant odor of the expired air whatever the origin may be. Oral malodor specifically refers to such odor originating from the oral cavity itself [9].

Halitosis, which means foul breath, might be interrelated to physiologic and/or pathologic reasons such as ear nose- throat diseases (chronic sinusitis, tonsillitis), gastrointestinal system diseases, diabetes mellitus, and acute rheumatic fever.

Also, more frequently, halitosis can be related to intraoral factors, including especially gram-negative anaerobic microorganisms on the dental plaque, in the periodontal pockets, in saliva, and on the dorsum of the tongue.
The volatile sulfur components (VSCs) consisting of hydrogen sulfide, dimethyl sulfide, and especially methyl mercaptan releasing through the proteolytic degradation of saliva, exfoliated epithelium, food debris, gingival crevicular fluid, plaque, postnasal drip, sulfurcontaining amino acids, and peptides in the blood by the anaerobic microorganisms found in the oral cavity are effective on the formation of halitosis.

The levels of VSCs in the mouth are also affected by to the number and depth of periodontal pockets, the bleeding tendency of periodontal pockets, and the amount of coating on the tongue [10].

\section{Etiology of halitosis}

Morning breath (physiologic halitosis) is caused by stagnation of saliva, entrapment of food particles, and presence of bacteria on the dorsum of the tongue due to the decrease in salivary movement during sleep [11].

\section{Dental origin (intraoral) $-75 \%$ of all cases}

Periodontal /peri-implant problem

Tooth problem

Soft-tissue problems

Dry mouth problems

Medical origin (extraoral) - $20 \%$ of all cases

Gastrointestinal disorders

Respiratory disorders

Immunocompromised

Autoimmune disorders

Liver failure, renal failure

Hormone fluctuation: menstruation

Metabolic disorders

Imaginary halitosis or delusional halitosis is a condition in which a subject believes that his or her breath odor is offensive despite any verification by a clinician or confidant. In one study, $28 \%$ of patients complaining of bad breath did not show signs of bad breath [11].

\section{Halitosis originates from oral cavity}

Although halitosis has multifactorial origins, the source of $90 \%$ cases is oral cavity. In oral cavity, temperatures may be reached up to $37^{\circ} \mathrm{C}$ (and changed between 34 and $37^{\circ} \mathrm{C}$ ). During exhaling also humidity may be reached up to $96 \%$ (and changed between $91 \%$ and $96 \%$ ) in oral exhalations. These conditions may afford a suitable environment for bacterial growth. The number of bacterial species, which are found in oral cavity, are over 500, and most of them are capable to produce odorous compounds which can cause halitosis. In these conditions, poor oral hygiene plays a key factor for multiplication of halitosis causative bacteria and causes an increase in halitosis.

These bacteria include especially Gr-negative species and proteolytic obligate anaerobes and they mainly retained in tongue coating and periodontal pockets. Among healthy individuals, with no history of halitosis and no periodontal diseases, some show halitosis because of retention of bacteria on the tongue surface. These bacteria degrade organic substrates (such as glucose, mucins, peptides, and proteins present in saliva, crevicular fluid, oral soft tissues, and retained 
debris) and produce odorous compounds. By the poor oral hygiene, food debris and dental bacterial plaque accumulate on the teeth and tongue, and cause caries and periodontal diseases like gingivitis and periodontitis [11].

The inflammation of gingival and periodontal tissues produces typical sources for oral malodors and plaque-related periodontal disease can increase the severity of halitosis.

However, the other forms of periodontal disease, especially acute and aggressive forms such as acute necrotizing ulcerative gingivitis, pericoronitis, Vincent's disease or aggressive forms of periodontitis, can increase unpleasant breath odor. The type of gingival enlargement which is dependent on inflammation or drugs (such as phenytoin, cyclosporine or calcium channel blockers) may increase the risk of bad odor. The severity of halitosis is affected from periodontal conditions, also periodontal conditions are affected by halitosis. The previous studies showed a relationship between oral halitosis and periodontal disease. Periodontal diseases may be developed by the volatile sulfurcontaining compound transition to periodontal tissues. However, it is still not well understood what is the relationship between periodontal health and oral malodors. Aylıkcı and Çolak: Halitosis: From diagnosis to management.

Besides periodontal conditions, untreated deep carious lesions also create the retention area for food debris and dental bacterial plaque and may cause halitosis. Another important factor in halitosis is the flow of saliva. The intensity of sulfur compounds is increased because of salivary flow reduction or xerostomia. Saliva functions as a buffering or a cleaning agent and keeps bacteria at a manageable level in the mouth. Reduction of the salivary flow has negative effects on self-cleaning of the mouth and inadequate cleaning of the mouth causes halitosis [11].

Reduction of Salivary flow may be affected from many reasons such as medications (e.g., antidepressants, antipsychotics, diuretic, and antihypertensive), salivary gland diseases (e.g., diabetes, Sjorgen's syndrome), chemotherapy, or radiotherapy. Other factors that contribute to halitosis are endodontic, surgical, and pathologic factors such as exposed tooth pulps and non-vital tooth with fistula draining into the mouth, oral cavity pathologies, oral cancer and ulcerations, extractions/healing wounds or prosthetics or dentition factors such as orthodontic fixed appliances, keeping at night or not regularly cleaning dentures, restorative crowns which are not well adapted, noncleaning the bridge body, and interdental food impaction. All these factors cause food or plaque retention area, raising bacterial amount, tissue breakdown, putrefaction of amino acids, and decreasing of saliva flow. All these conditions result in the release of volatile compoundsand cause halitosis [11].

\section{Halitosis originates from non-oral sources}

Nearly $8 \%$ of the halitosis cases caused from an extraoral source. This type halitosis has many sources, but it is rarely seen. Respiratory system problems, gastrointestinal disease, hepatic disease, hematological or endocrine system disorders and metabolic conditions can all be the causes of halitosis. Respiratory system problems can be divided into upper and lower respiratory tract problems. They are sinusitis, antral malignancy, cleft palate, foreign bodies in the nose or lung, nasal malignancy, subphrenic abscess, nasal sepsis, tonsilloliths, tonsillitis, pharyngeal malignancy, lung infections, bronchitis, and bronchiectasis lung malignancy. Bacterial activity in this pathology causes halitosis which leads to putrefaction of the tissues or causes tissue necrosis and ulcerations and production of malodorous gases, which are expired causing halitosis. [Gastrointestinal diseases cause halitosis. Pyloric stenosis, duodenal obstruction, aorto-enteric anastomosis, pharyngeal pouches, zenker's diverticulum, hiatal hernia cause food retention. Reflux esophagitis, achalasia, steatorrhea, or other malabsorption syndromes may cause excessive flatulence or Helicobacter pylori infection causes gastric ulcers and VSC levels increase in oral breath. Levels of VCS's in oral breath may be higher in patients with erosive than non erosive oesophagogastro-duodenal mucosal disease although VSC levels are not influenced by the degree of mucosal damage [11].

Also, hepatic or hematological diseases which are hepatic failure (foetorhepaticus) and leukemia's, renal failure (usually end-stage renal failure), endocrine system disorders which are diabetic ketoacidosis or menstruation (menstrual breath), metabolic disorder which are trimethylaminuria and hypermethioninemia may cause halitosis [11].

\section{Other causes of halitosis}

Dietary products such as garlic, onions, spiced foods cause transient unpleasant odor or halitosis. Therewithal drugs such as alcohol, tobacco, betel, solvent abuse, chloral hydrate, nitrites and nitrates, dimethyl sulfoxide, disulphiram, somecytotoxics, phenothiazines, amphetamines, suplatast tosilate, and paraldehyde may create the same effect [11].

\section{Assessment of halitosis}

There are three generally accepted methods for the assessment of oral malodor: organoleptic measurement. (Figure 1) gas chromatography (GC), and portable sulfide monitoring. (Figure 2). A portable Halimeter (Halimeter, Interscan Corp., Chatsworth, CA) [12]

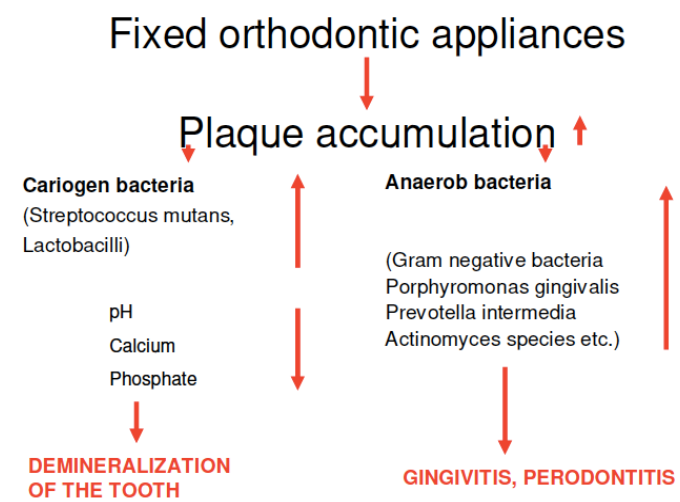

OF THE TOOTH

Figure 1. Risk of treatment with orthodontic appliances

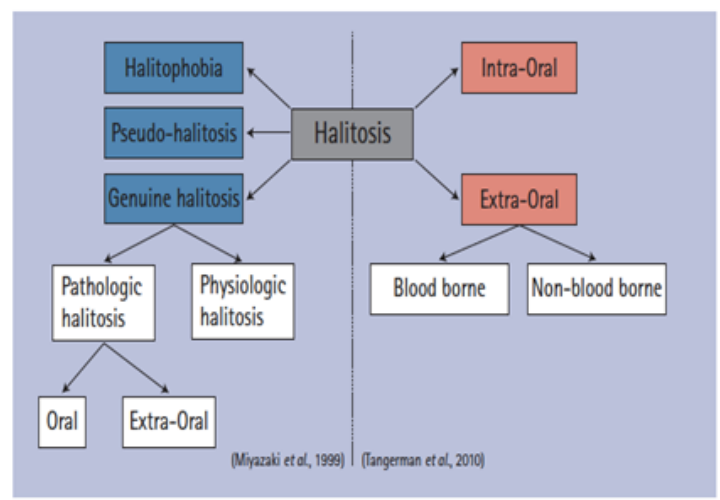

Figure 2. Two previous classifications. Miyazaki et al.1999 is generally the most widely used 


\section{Review of Literature}

Halitosis is a common condition, affecting around $25 \%$ of the general population. The origin of the problem largely arises from intraoral causes, whereas only a limited number of cases are the result of extra oral or systemic problems. Nevertheless, proper investigation and management of these extra oral causes is important for the total understanding of this phenomenon. Halitosis from an extra-oral origin can be the sign of an underlying systemic disease. Therefore, it is substantiated to organize halitosis consultations in a multidisciplinary setting, assembling periodontologists , ENT specialists, specialists in internal medicine and psychologists or even psychiatrists. Although oral malodor is mostly associated with poor oral hygiene and the presence of gingivitis or even periodontitis, evidence suggests that anaerobic microorganisms present in the tongue coating, are the overwhelming cause of this condition. A limited number of successful treatment regimens have been described, but more research on the longterm outcomes of these therapies will be required. Also new and more long lasting in-office treatments should be developed and tested [13].

Fixed orthodontic therapy is a risk factor for malodor at 1 week after bonding, independent of the change in plaque index (PI). Selfligating brackets (SLBs) systems controlled malodor slightly better than conventional brackets (CB) systems, significantly at the first week after bonding and, thus, could be considered for orthodontic patients at high risk for malodor [10].

Orthodontic treatment affects oral malodor with regard to the PI, gingival index (GI), and periodontal pocket depth (PPD). The critical limit of oral malodor was reached at the end of 7 months. Oral malodor can be used as an indicator to evaluate the oral health of patients.

Clinically, after 7 months of fixed orthodontic treatment, the clinician will follow not only the GI and PI, but also the oral malodor to establish ideal oral health in patients [14] the study Reduction of Oral Levels of Volatile Sulfur Compounds (VSC) by Professional Toothcleaning and OralHygiène Instruction in Non-halitosis Patients reveals that in a group of patients without bad breath, an oral hygiene training program with professional tooth cleaning, motivation and instruction in self-applied oral hygiene procedures is capable of reducing both papillary bleeding and oral levels of VSC Halimeter readings over the observation period of four weeks. On the basis of these findings, further studies should elucidate whether Halimeter ${ }^{\circ}$ readings might be used to elevate patient motivation to improve their oral hygiene levels [10].

SLBs positively affected halitosis and periodontal status but did not alter microbial colonization.

CBs led to an increase in halitosis with the accretion of plaque accumulation. Therefore, these brackets with elastomeric ligatures are not recommended for use in patients with poor oral hygiene or individuals prone to bad breath. SLBs could be considered for enabling better oral health and hygiene [14].

The SLBs do not have an advantage over CBs with respect to periodontal status and halitosis .Rather than periodontal status, the tongue coating might have more effect on halitosis [10].

Oral malodor reached the critical level during fixed orthodontic treatment. The PI and GI scores increased immediately after bonding. Oral malodor could be another indicator to evaluate oral health and to warn patients to achieve ideal oral hygiene [14] the measurement of the VSC levels detected by portable devices can be used as an adjuvant tool with OT in subjects without malodor complaint. In spite of the great improvement of such devices in the last years, OT remains the "gold standard" method for the diagnosis of bad breath.

Halitosis in non-complainer patients at the same level as the organoleptic examination. In addition, they cannot be considered reliable even when their results are gathered and analyzed together. Even though the use of the portable devices can lead to a considerable number of negative results, their value must be highlighted as an important tool for malodor screening.

Finally, these devices can be also very useful for patient's followup and to differentiate distinct types of halitosis, such as those caused by great amount of sulphur compounds from others (i.e. organic volatilecompounds) [15]. A statistically significant increase on the plaque and tongue coating indices was observed over the course of the investigation, as well as statistically significantly increased values in organoleptic measurements. A positive correlation between fixed orthodontic appliances and the occurrence of halitosis was established. However, it remains to be seen how the parameters under investigation were to behave in a larger study of more participants and a longer period of monitoring. It is entirely possible for oral hygiene to improve with time and that the factors determining the ecosystem of the oral cavity were subsequently modified.

The occurrence of halitosis during orthodontic treatment involving fixed orthodontic appliances is an indicator of oral health for practitioners. Organoleptic measurements can easily be performed during conversation with the patient and during clinical consultation. Because of the socially and professionally inhibiting nature of halitosis, this study's results are relevant insofar as they might serve as motivating factors for patients to dedicate themselves to maintaining adequate oral hygiene [16].

\section{Materials and methods}

\section{Study design}

A single-blinded randomized controlled trial was done at orthodontic clinics within three branches of the Riyadh Elm University hospital, Riyadh, Saudi Arabia.

The study protocol was approved by the institutional review board at Elm University, Riyadh, Saudi Arabia. The protocol was also registered at Clinicaltrials.gov with registration number:PGRP/43734003/316

\section{Study population}

The ethical approval has been obtained from the International Review Board (IRB) of Riyadh Elm University.

Informed consent was obtained for all participants or their parents if the patient was under 18 years old. each participant was randomly assigned to one of four groups using simple randomization technique on a computer program. a data analyzer generated the random sequence.

The volunteers and controls for this study participated between December 2018 and April 2019.

\section{Inclusion criteria}

Participants under fixed orthodontic appliance treatment.

Participants did not have mental or physical disabilities.

Participants were willing to comply with given oral hygiene instructions. 


\section{Exclusion criteria}

- Antibiotic use during or up to 4 months prior to the study.

- Smoking, having an orthognathic surgery requirement

- Self-declared history of systemic diseases

- An unwillingness to participate in the study.

\section{Sample}

Recruitment of participants was initially started on $2^{\text {nd }}$ December 2018. Each participant was recruited and underwent screening for inclusion. The participants who met the inclusion criteria were examined using the outcome measures specified below, re-examined after 4-6 weeks and re-examined again after 8-12 weeks. The final data collection took place on $4^{\text {th }}$ April 2019.

Sample size calculation has been done based on previous literature. The sample size was calculated as 39 participants.

Informed consent was obtained for all participants or their parents if the patient was under 18 years old. Each participant was randomly assigned to one of four groups using simple randomization technique on a computer program. A data analyzer generated the random sequence. Allocation was concealed by having patients withdraw opaque envelops from a container and then, took envelops to one independent coordinator who then, checked to which group the patient was assigned. Examiners, data collectors, and analyzers were blinded in regard to patient group throughout the study. Blinding of examiners was practiced as the examiners were only allowed to enter the clinic and perform clinical examination without knowing or interacting with patients. Blinding patients was not applicable.

\section{Group I}

Patients were instructed to use their manual tooth brush and dental floss twice daily.

\section{Group II}

Patients were given electric tooth brush and were instructed to use it along with dental floss twice daily.

\section{Group III}

Patients were given a water jet device and instructed to use it twice daily along with their manual tooth brush.

\section{Group IV}

The patients were given an electrical tooth brush and water jet device and instructed to use them twice daily.

\section{Reliability testing}

Two examiners carried out examinations. They were calibrated by having each one of them practicing PI and GI on 20 participants and comparing their scores using interclass correlation coefficient.

\section{Orthodontic treatment}

Orthodontic therapy consisted of molar bands with edgewise triple buccal tubes with vertical hooks. also, in the second and first premolars, canines, and lateral and central incisors, direct-bonded brackets (MBT) were used. (MBT) is orthodontic bracket system refered to the BennettMclaughlin et al.

The measurements were taken while the patients had stainless steel arch wires.
The therapy was applied by the orthodontic residents at R.E.U. clinics to all patients.

\section{Oral malodor}

In this study, a portable halimeter (Tanita Breath Checker HC312SF Fitscan ) was used to detect the oral malodor.

Each patient kept their mouth closed for $60 \mathrm{~s}$ prior to the measurement.

The portable halimeter was placed in front of patients mouths with a distance of 4 inches a patient was instructed to blow air.

The participant was asked not to inhale air during the halimeter reading.

Measurements were duplicated and the mean values were calculated.

Oral malodor values were divided into five categories and classified as normal (1), weak (2), medium (3) strong (4), or very strong (5).

\section{Tanita Breath Checker HC-312SF Fitscan}

Is an innovative palm size monitor that detects and measures the presence of breath odors (VSCS).

It measures odor in seconds and results are displayed similar to organoleptic method.

\section{Statistical analysis}

Descriptive statistics were calculated and Kruskal Wallis test was used to determine differences between groups at each time point.

\section{Results}

Thirty-nine subjects $13-38$ years of age (mean 21.84 years) with fixed orthodontic appliances in both arches were randomized in a randomized controlled trial with four parallel arms to test groups in a triple-blinded study, with thirty-nine participants completing all study visits. Mean age of patients was $21.8462 \pm 5.29915$ years and females were slightly more than males ( $\mathrm{n}$ of females $=56.4 \%$ and $\mathrm{n}$ of males $=$ $43.6 \%$ ) (Table 1 ).

Descriptive statistics showed the sample included males (43.6\%) and females (56.4\%). Gender and age distribution is shown in Table 1. Means and SD for halitosis for all groups at each time point is shown in (Table 2).

There was a statistically significant difference in halitosis between groups in $\mathrm{T} 1$ and $\mathrm{T} 3(\mathrm{p}=0.049, \mathrm{p}=0.036)$ (Table 3$)$. The differences at each time point showed that there is a statistically significant difference in T1 between $\mathrm{G} 1$ (mean=04.22, SD=01.09291) and G2 (mean=04.50, $\mathrm{SD}=0.849)(\mathrm{p}=0.049)$ Table 4 shows the differences between groups at $\mathrm{T} 1$.

The mean age of females were more than males Figure 1 (mean of males $=22.1176 \pm 5.91484$ and mean of females $=21.6364 \pm 4.90428)$.

\section{Discussion}

This study was conducted to evaluate the effects of different teeth cleaning methods on the level of volatile Sulphur compounds (VSC)

Table 1. Participants age values

\begin{tabular}{|c|c|c|c|c|c|c|}
\hline Gender & Mean & N & $\begin{array}{c}\text { Std. } \\
\text { deviation }\end{array}$ & Minimum & Maximum & $\begin{array}{c}\text { \% of total } \\
\text { N }\end{array}$ \\
\hline Male & 22.1176 & 17 & 5.91484 & 14.00 & 35.00 & $43.6 \%$ \\
\hline Female & 21.6364 & 22 & 4.90428 & 14.00 & 30.00 & $56.4 \%$ \\
\hline total & 21.8462 & 39 & 5.29915 & 14.00 & 35.00 & $100.0 \%$ \\
\hline
\end{tabular}


Table 2. Value of group T1,T2,T3

\begin{tabular}{|c|c|c|c|c|}
\hline \multicolumn{5}{|l|}{ Report } \\
\hline \multicolumn{2}{|l|}{ Group } & \multirow{2}{*}{\begin{tabular}{|l} 
HalitosisT1 \\
4.2222
\end{tabular}} & \multirow{2}{*}{$\begin{array}{l}\text { HalitosisT2 } \\
3.3000\end{array}$} & \multirow{2}{*}{$\begin{array}{l}\text { HalitosisT3 } \\
3.5000\end{array}$} \\
\hline \multirow{5}{*}{$\begin{array}{l}\text { Manual toothbrush }+ \text { super } \\
\text { Floss }\end{array}$} & Mean & & & \\
\hline & $\mathrm{N}$ & 9 & 10 & 10 \\
\hline & Std. Deviation & 1.09291 & 1.33749 & 1.17851 \\
\hline & Minimum & Weak & Weak & Weak \\
\hline & Maximum & Very Strong & Very Strong & Very Strong \\
\hline \multirow{5}{*}{ Manual toothbrush +Waterjet } & Mean & 4.2727 & 4.5000 & 4.8000 \\
\hline & $\mathrm{N}$ & 11 & 10 & 10 \\
\hline & Std. Deviation & 1.10371 & 0.84984 & 0.42164 \\
\hline & Minimum & Weak & Medium & Strong \\
\hline & Maximum & Very Strong & Very Strong & Very Strong \\
\hline \multirow{5}{*}{$\begin{array}{l}\text { Electric toothbrush }+ \text { super } \\
\text { Floss }\end{array}$} & Mean & 2.8000 & 3.5000 & 3.5000 \\
\hline & $\mathrm{N}$ & 10 & 10 & 10 \\
\hline & Std. Deviation & 1.54919 & 1.64992 & 1.35401 \\
\hline & Minimum & Normal & Normal & Normal \\
\hline & Maximum & Very Strong & Very Strong & Very Strong \\
\hline \multirow{5}{*}{ Electric toothbrush + Waterjet } & Mean & 3.1250 & 3.6250 & 3.8750 \\
\hline & $\mathrm{N}$ & 8 & 8 & 8 \\
\hline & Std. Deviation & 1.45774 & 1.18773 & 1.12599 \\
\hline & Minimum & Normal & Weak & Weak \\
\hline & Maximum & Very Strong & Very Strong & Very Strong \\
\hline \multirow{5}{*}{ Total } & Mean & 3.6316 & 3.7368 & 3.9211 \\
\hline & $\mathrm{N}$ & 38 & 38 & 38 \\
\hline & Std. Deviation & 1.42224 & 1.32918 & 1.17131 \\
\hline & Minimum & Normal & Normal & Normal \\
\hline & Maximum & Very Strong & Very Strong & Very Strong \\
\hline
\end{tabular}

Table 3. Hypothesis Test Summary

\begin{tabular}{|c|c|c|c|c|}
\hline \multicolumn{5}{|c|}{ Hypothesis Test Summary } \\
\hline & Null Hypothesis & Test & Sig. & Decision \\
\hline 1 & $\begin{array}{l}\text { The distribution of } \\
\text { HalitosisT1 } 1 \text { is the same } \\
\text { across categories of } \\
\text { Group. }\end{array}$ & $\begin{array}{l}\text { Independent-Samples } \\
\text { Kruskal-Wallis Test }\end{array}$ & 0.049 & $\begin{array}{l}\text { Reject the null } \\
\text { hypothesis. }\end{array}$ \\
\hline 2 & $\begin{array}{l}\text { The distribution of } \\
\text { HalitosisT2 is the same } \\
\text { across categories of } \\
\text { Group. }\end{array}$ & $\begin{array}{l}\text { Independent-Samples } \\
\text { Kruskal-Wallis Test }\end{array}$ & 0.188 & $\begin{array}{l}\text { Retain the null } \\
\text { hypothesis. }\end{array}$ \\
\hline 3 & $\begin{array}{l}\text { The distribution of } \\
\text { HalitosisT3 is the same } \\
\text { across categories of } \\
\text { Group. }\end{array}$ & $\begin{array}{l}\text { Independent-Samples } \\
\text { Kruskal-Wallis Test }\end{array}$ & 0.036 & $\begin{array}{l}\text { Reject the null } \\
\text { hypothesis. }\end{array}$ \\
\hline
\end{tabular}

Table 4. Comparison between each two groups

\begin{tabular}{|l|c|c|}
\hline Differences between groups & Mean Difference & p-value \\
\hline $\begin{array}{l}\text { (Electric toothbrush + Superfloss) - } \\
\text { (Electric toothbrush + Waterjet) }\end{array}$ & -0.325 & 0.696 \\
\hline $\begin{array}{l}\text { (Electric toothbrush + Superfloss) - } \\
\text { (Manual toothbrush + Superfloss) }\end{array}$ & -1.422 & 0.039 \\
\hline Electric+Floss-Manual+Waterjet & -1.473 & 0.021 \\
\hline $\begin{array}{l}\text { (Electric toothbrush + Waterjet) - (Manual } \\
\text { toothbrush + Superfloss) }\end{array}$ & -1.097 & 0.116 \\
\hline Electric+Waterjet-Manual toothbrush +Wjet & -1.148 & 0.076 \\
\hline $\begin{array}{l}\text { (Manual toothbrush + Superfloss) - } \\
\text { (Manual toothbrush + Waterjet) }\end{array}$ & -0.051 & 0.891 \\
\hline a. Significance values have been adjusted by the Bonferroni correction for multiple tests.
\end{tabular}

a. Significance values have been adjusted by the Bonferroni correction for multiple tests.

by portable halimeter (Tanita Breath Checker HC-312SF Fitscan). Participants were divided into four groups; Group A: manual toothbrushes with super floss, Group B: electric toothbrushes with super floss, Group C: manual toothbrushes with water flosser, Group
D: electric toothbrushes with water flosser. Measurements were taken at baseline, after 4-6 weeks, and then after 8-12 weeks.

In this study, (VSC) level were detected using portable halimeter, this choice of the device was based on the validity, simplicity and reliability of this device. It has also been used by [17].

There was a statistically significant difference in halitosis between groups in $\mathrm{T} 1$ and $\mathrm{T} 3(\mathrm{p}=0.049, \mathrm{p}=0.036)$ (Table 3$)$. The differences at each time point showed that there is a statistically significant difference in T1 between G1 (mean=04.22, $\mathrm{SD}=01.09291)$ and $\mathrm{G} 2$ (mean=04.50, $\mathrm{SD}=0.849)(\mathrm{p}=0.049)$.

In contrast, other similar studies have chosen other methods and devices such as organoleptic measurement by [18]. A portable Halimeter (Halimeter, Interscan Corp., Chatsworth, CA, USA) [12], breath alert assessment by $[19,20]$ The Oral Chroma portable gas chromatograph by [21].

Table 5. Pairwise Comparisons of Group t3

\begin{tabular}{|l|c|c|}
\hline \multicolumn{2}{|l|}{ Pairwise Comparisons of Group } & Mean \\
\hline Sample 1-Sample 2 & 0.000 & 0.907 \\
\hline $\begin{array}{l}\text { Manual toothbrush +super Floss-Electric } \\
\text { toothbrush +super Floss }\end{array}$ & -0.375 & 0.522 \\
\hline $\begin{array}{l}\text { Manual toothbrush+ super Floss-Electric } \\
\text { toothbrush+waterjet }\end{array}$ & -1.300 & 0.010 \\
\hline $\begin{array}{l}\text { Manual toothbrush +super Floss-Manual } \\
\text { toothbrush+waterjet }\end{array}$ & -0.375 & 0.596 \\
\hline $\begin{array}{l}\text { Electric toothbrush +super Floss-Electric } \\
\text { toothbrush+waterjet }\end{array}$ & -1.300 & 0.014 \\
\hline $\begin{array}{l}\text { Electric toothbrush +super Floss-Manual } \\
\text { toothbrush+waterjet }\end{array}$ & -0.925 & 0.073 \\
\hline $\begin{array}{l}\text { Electric toothbrush +waterjet -Manual } \\
\text { toothbrush+waterjet }\end{array}$ & \\
\hline
\end{tabular}

Each row tests the null hypothesis that the Sample 1 and Sample 2 distributions are the same. Asymptotic significances (2-sided tests) are displayed. The significance level is .05.

a. Significance values have been adjusted by the Bonferroni correction for multiple tests.

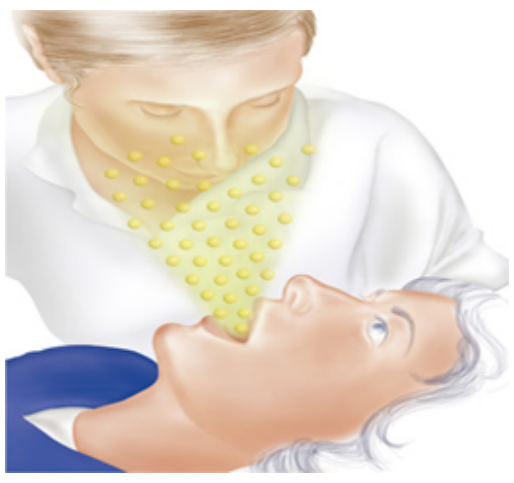

Figure 3. Organoleptic measurement

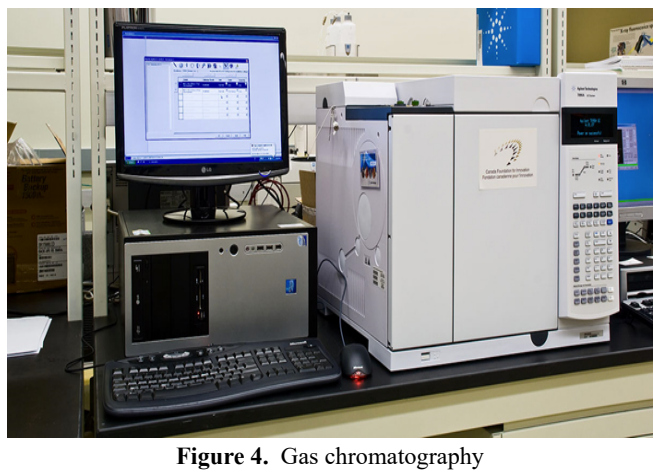

Volume 5: 6-8 


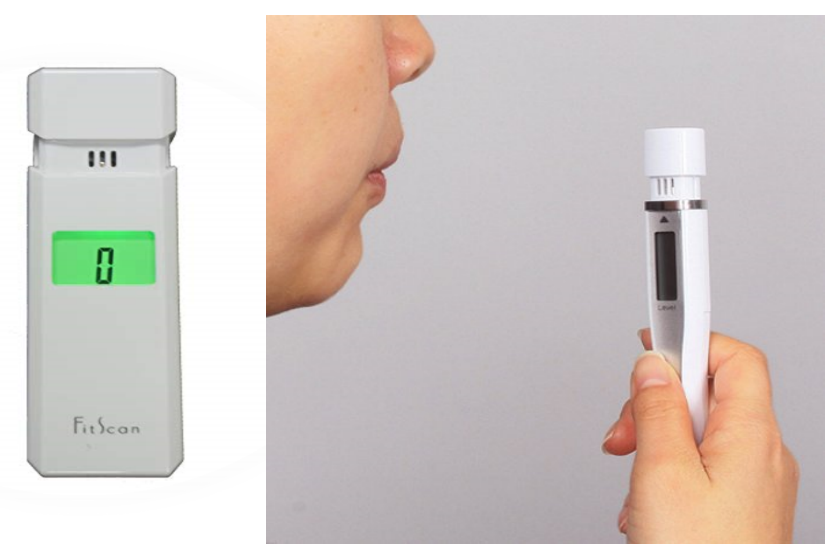

Figure 5. Tanita Breath Checker HC-312SF Fitscan

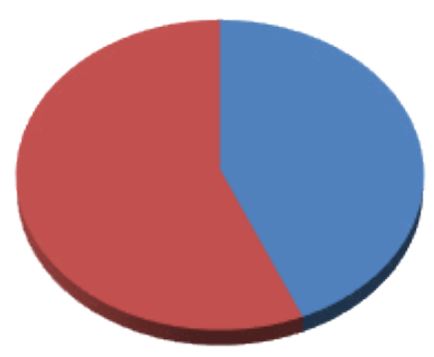

Gender distribution of the sample

$1 \square 2$

Figure 6. The mean age of females and males

Recently, halitosis has received much more scientific attention. The exact pathophysiological mechanism of halitosis is unclear, but all studies now agree $85-90 \%$ of cases are of intra oral origin tongue coating and periodontal disease are the main causes and VSC are the major compounds that contribute to oral malodor. Nevertheless, probably due to the numerous factors that can influence a systemic cause, there is much more controversy regarding the nature of systemic origin. Most of the studies declare that extra oral causes are predominantly found in the ear, nose and throat regions or in rare cases in the gastrointestinal tract, but metabolic disorders like diabetes, liver pathology, endocrinological diseases, medications, mouth breathing or stressful situations have been studied as causes or possible contributions that increase halitosis [22].

More studies have to be done to evaluate the differences between gender and different age groups patients with fixed orthodontic appliances [23].

The results showed that patients brush their teeth regularly, the majority twice a day, but with only a small percentage of respondents reporting that they brush their teeth three or more times a day. The respondents claimed that their oral hygiene features changed after they started orthodontic treatment. According to the studies of other authors, after the beginning of orthodontic treatment the accumulation of plaque increases and it is difficult to remove plaque from around brackets and interdentally. Because of changes in microflora, the risks of enamel demineralization, periodontal disease and halitosis also increase. It is important to maintain good oral health and to use additional oral hygiene measures [23]. The study showed that adolescents rarely use additional oral hygiene measures such as an interdental toothbrush, dental floss or an oral irrigator, and both during and after orthodontic treatment are faced with complications such as plaque accumulation, enamel demineralization, tooth discoloration and calculus. To avoid complications, it is important to use an orthodontic or electric toothbrush, fluoride-containing measures, an interdental toothbrush or dental floss [23].

Oral malodour originates mainly from the mouth itself while the malodourous substrates are most commonly associated with microbial metabolism. The oral surfaces are colonized by large number of bacterial species which are known to produce malodourous compounds. However, no obvious association exists between halitosis and any specific bacterial infection, suggesting that bad breath reflects complex interactions between several oral bacterial species. Most of the major malodourous compounds are Volatile Sulphur Compounds (VSCs) such as hydrogen sulphide (H2S), methylmercaptan (CH3SH) and dimethyl sulphide ((CH3)2S). In vitro and in vivo studies have demonstrated that oral gram negative anaerobic bacteria such as Porphyromonas Gingivalis (P.G.), Fusobacterium Nucleatum (F.N.), Treponema Denticola (T.D.) and several species of other oral bacteria can produce VSCs. These bacteria can be isolated from the subgingival plaque in gingivitis or periodontitis patients, and from the saliva and the dorsum of the tongue in healthy subjects. Odour outcomes are significantly correlated with total counts of bacteria and the diversity of each type [23].

Several microorganisms recovered from periodontal lesions of gingivitis and periodontitis can produce large amounts of VSCs. The bacterial interactions are most likely to occur in the gingival crevices and periodontal pockets, but oral malodour can also arise from the posterior dorsal surface of the tongue. A consequence of its large and irregular surface is that it is an ideal niche for oral bacteria. Since desquamating epithelial cells and remnants are available, putrefaction occurs.

During the process of bacterial putrefaction, however, compounds other than sulphur compounds are also formed. Peptides are hydrolysed to aminoacids which can be metabolized further to amines or polyamines. Even when the contribution of all of these compounds to oral malodour has not been found, other studies have clearly demonstrated a significant correlation between some of them and oral malodour. The researchers concluded that halitosis is a result of multifaceted interactions between diverse species of bacteria. Other oral pathological sources that have been suggested as a cause of halitosis are dental cavities, exposed tooth pulps, healing wounds, interdental food impaction, dentures kept in at night or not regularly cleaned, fixed orthodontic appliances, restorative crowns that are not well adapted, cysts with fistula draining into the mouth, peri-implantitis, pericoronitis, oral cancer, ulcerations and factors causing a decreased salivary flow rate [23].

Saliva plays a central role in the development of bad breath. Patients with xerostomia have an increase level of plaque on their teeth and tongue, the antimicrobial activity of the saliva disappears and there is a transition from gram-positive to gram-negative species. There are a number of clinical conditions that can cause changes to salivary patterns. One such condition is a change from nasal to mouth breathing, which causes adaptive changes drying the mucosa . Albuquerque et al. evaluated halitosis and sialometry in patients submitted to head and neck radiotherapy, because of the radiosensitivity, the salivary glands had a decreased potential of saliva production. The results showed a strong association between hyposalivation and halitosis. This study and the many causes of xerostomia, show it is important to find out and eliminate the cause of hyposalivation in the treatment of halitosis.

Several studies reflect the importance of carrying out the appropriate measures under the right conditions in order to have the most reliable 
results. It would be difficult to ascertain which factors produced the strongest evidence of halitosis as it would entail a control to avoid contamination from other external sources some of which may have been consumed some time ago which may obscure the results, such as the use of healthcare products, consumption of alcohol, tobacco and food [22].

\section{Limitation of this study}

Decrease number of samples .

Bacteria on the dorsum of the tongue not assessed.

This study was done on patients with good oral hygiene, it might be necessary to do other future studies with poor oral hygiene patients.

\section{Conclusion}

When Comparing the efficacy of these four different methods of teeth cleaning on reducing VSC in patients with active orthodontic fixed appliances, results found that there are No significant difference between controlled group and the other groups

\section{Future studies recommendation}

Additional studies of longer duration and larger sample size are recommended to evaluate the efficacy and compliance of the 4 oral hygiene methods.

\section{References}

1. Kaygisiz E, Uzuner FD, Yuksel S, Taner L, Çulhaoğlu R, et al. (2015) "Effects of SelfLigating and Conventional Brackets on Halitosis and Periodontal Conditions." Angle Orthod 85: 468-473. [Crossref]

2. Nalçacı R, Sönmez Is (2008) Evaluation of oral malodor in children. Oral Surgery, Oral Medicine, Oral Pathology, Oral Radiology, and Endodontology 106: 384-388.

3. Quirynen M, Zhao H, Van Steenberghe D (2002) Review of the treatment strategies for oral malodour. Clin Oral Investig 6: 1-10. [Crossref]

4. Ileri Keceli T, Gulmez D, Dolgun A, Tekcicek M, Huang J, et al. (2015) The relationship between tongue brushing and halitosis in children: A randomized controlled trial. Oral Diseases 21: 66-73.

5. Aylikcı BU (2013) Halitosis: From diagnosis to management. 4: 14-23.

6. Bunzel M, Ralph J, Marita JM, Hatfield RD, Steinhar, H (2001) Diferulates as structural components in soluble and insoluble cereal dietary fibre. J SCI FOOD AGR 81: 653660.
7. Tangerman A (2002) Halitosis in medicine: a review. Int dent J 52: 201-206. [Crossref]

8. Çiçek Y, Orbak R, Tezel A, Orbak Z, Erciyas K (2003) Effect of tongue brushing on oral malodor in adolescents. Pediatr Int 45: 719-723. [Crossref]

9. Sekino S, Ramberg P, Uzel Ng, Socransky S, Lindhe J (2003) Effect of various chlorhexidine regimens on salivary bacteria and de novo plaque formation. $J$ CLIN PERIODONTOL 30: 919-925.

10. Kaygisiz E, Uzuner Fd, Yuksel S, Tanerd L, Culhaołlu R, et al. (2016) The influence of fixed orthodontic appliances on halitosis. BMC Oral Health 21: 483-490.

11. Aylıkcı Bu, Çolak H (2013) Halitosis: From diagnosis to management. J Nat Sci Biol Med 4: 14. [Crossref]

12. Sökücü O, Akpınar A, Özdemir H, Birlik M, Çalışır M (2016) "The Effect of Fixed Appliances on Oral Malodor from Beginning of Treatment till 1 Year." BMC Oral Health pp: 164.

13. Bollen Curd ML, Beikler T (2012) "Halitosis: The Multidisciplinary Approach." Int J Oral Sci pp. 55-63. [Crossref]

14. Babacan H, Sokucu O, Marakoglu I, Ozdemir H, Nalcaci R (2011) Effect of fixed appliances on oral malodor. Am J Orthod Dentofacial Orthop 139: 351-355. [Crossref]

15. Miranda PC, Xvhg R, Folqlfdo LQ, Qrw S, Vwxglhv PDQ, et al. (2017) Assessment of the accuracy of portable monitors for halitosis evaluation in subjects without malodor complaint . Are they reliable for clinical practice? J Appl Oral Sci 25: 559-565. [Crossref]

16. Zurfluh MA, Van Waes Hjm, Filippi A (2013) The influence of fixed orthodontic appliances on halitosis. Research and Science 123: 1064-75. [Crossref]

17. Author HC (2018) Advanced Diagnostic Aids For Oral Malodour Detection - A Review Periodontics. International Journal Of Scientific Research pp: 72-75.

18. Feres M, Figueiredo LC, Faveri M, Guerra MC, Mateo LR, et al. (2015) The efficacy of two oral hygiene regimens in reducing oral malodour: A randomised clinical trial. International Dental Journal 65: 292-302.

19. Falcão D, Miranda P, Almeida T, Scalco M, Fregni F, et al. (2019) Assessment of the accuracy of portable monitors for halitosis evaluation in subjects without malodor complaint. Are they reliable for clinical practice?

20. Belongia EA, Simpson MD, King JP, Sundaram ME, Kelley NS, et al. (2016) Variable influenza vaccine effectiveness by subtype: a systematic review and meta-analysis of test-negative design studies. The Lancet Infectious Diseases 16: 942-951.

21. Tonzetich J (1971) Direct gas chromatographic analysis of sulphur compounds in mouth air in man. Arch Oral Biol 16: 587-597. [Crossref]

22. Monfort Codinach M, Chimenos Küstner E, Alburquerque R, López López J (2014) Update of intra and extra oral causes of halitosis: A systematic review. Oral Health and Dental Management p. 975-981.

23. Kudirkaite I, Lopatiene K, Zubiene J, Saldunaite K (2016) Age and gender influence on oral hygiene among adolescents with fixed orthodontic appliances. Stomatologija 18: 61-65.

Copyright: (C2020 AlHarbi AM. This is an open-access article distributed under the terms of the Creative Commons Attribution License, which permits unrestricted use, distribution, and reproduction in any medium, provided the original author and source are credited. 